\title{
OPEN Evaluation of endogenous miRNA reference genes across different zebrafish strains, developmental stages and kidney disease models
}

\author{
Florian Siegerist ${ }^{1,5}$, Tim Lange ${ }^{1,5}$, Anna lervolino ${ }^{1,2}$, Thor Magnus Koppe ${ }^{1}$, Weibin Zhou ${ }^{3}$, \\ Giovambattista Capasso ${ }^{2,4}$, Karlhans Endlich ${ }^{1}$ \& Nicole Endlich ${ }^{1 凶}$
}

The majority of kidney diseases arise from the loss of podocytes and from morphological changes of their highly complex foot process architecture, which inevitably leads to a reduced kidney filtration and total loss of kidney function. It could have been shown that microRNAs (miRs) play a pivotal role in the pathogenesis of podocyte-associated kidney diseases. Due to their fully functioning pronephric kidney, larval zebrafish have become a popular vertebrate model, to study kidney diseases in vivo. Unfortunately, there is no consensus about a proper normalization strategy of RT-qPCR-based miRNA expression data in zebrafish. In this study we analyzed 9 preselected candidates dre-miR92a-3p, dre-miR-206-3p, dre-miR-99-1, dre-miR-92b-3p, dre-miR-363-3p, dre-let-7e, dre-miR-454a, dre-miR-30c-5p, dre-miR-126a-5p for their capability as endogenous reference genes in zebrafish experiments. Expression levels of potential candidates were measured in 3 different zebrafish strains, different developmental stages, and in different kidney disease models by RT-qPCR. Expression values were analyzed with NormFinder, BestKeeper, GeNorm, and DeltaCt and were tested for inter-group differences. All candidates show an abundant expression throughout all samples and relatively high stability. The most stable candidate without significant inter-group differences was dre-miR-92b-3p making it a suitable endogenous reference gene for RT-qPCR-based miR expression zebrafish studies.

MicroRNAs (miRs) regulate protein expression by translational suppression via RNA interference. With their seed sequence they bind specifically to target mRNAs, thereby blocking translation and/or facilitating mRNA degradation ${ }^{1}$. In the recent past it could have been shown that microRNAs play a pivotal role in kidney development, physiology and pathology ${ }^{2-4}$.

Over the years, larval zebrafish have emerged a popular model organism, due to their small size, high reproductive potential and genetic accessibility ${ }^{5}$. The fact that zebrafish develop a fully filtrating glomerulus attached to a pair of tubules with high structural and molecular homology two days past fertilization (dpf) makes this model highly relevant for the investigation of human glomerular kidney diseases ${ }^{6}$. Most of these diseases arise from highly specialized glomerular cells, the podocytes. They are central players for glomerular permselectivity as they form the filtration slits together with neighboring podocytes. This is ensured by their complex interdigitating branching morphology. Interruptions in this morphology or the loss of podocytes subsequently leads to the development of nephrotic syndrome, a pathologic condition in which the size selectivity of the kidney filter gets impaired. Patients with nephrotic syndrome develop high-molecular weight proteinuria together with hypoproteinemic edema as typical characteristics ${ }^{7}$. A multitude of studies used larval zebrafish to model proteinuric glomerular diseases may it be with the help of morpholino-guided knockdown of podocyte genes ${ }^{8,9}$ or treatment with podocyte-directed drugs ${ }^{10-12}$. Exemplary for morpholino-guided gene knockdowns, we targeted two disease-causing genes for which zebrafish models have been established. First, wt $1 a$, a transcription factor essential for podocyte differentiation and maintenance ${ }^{8}$. Second, $n p h s 1$, the gene encoding nephrin, a cell-cell contact protein that is the important for glomerular filtration barrier function and which absence in patients leads to steroid-resistant nephrotic syndrome.

\footnotetext{
${ }^{1}$ Institute of Anatomy and Cell Biology, University Medicine Greifswald, Friedrich-Loeffler-Str. 23c, 17487 Greifswald, Germany. ${ }^{2}$ Biogem Research Institute Gaetano Salvatore, Ariano Irpino, Italy. ${ }^{3}$ Division of Nephrology, Department of Medicine, Icahn School of Medicine at Mount Sinai, New York City, NY, USA. ${ }^{4}$ Department of Translational Medical Sciences, University of Campania "L. Vanvitelli", Naples, Italy. ${ }^{5}$ These authors contributed equally: Florian Siegerist and Tim Lange. ${ }^{\circledR}$ email: nicole.endlich@uni-greifswald.de
} 
The relevance of miRs for glomerular pathophysiology has been proven several times. Studies have shown that the podocyte-specific knockout of the miR-processing ribonucleases Dicer and Drosha leads to proteinuria and glomerulopathy ${ }^{13-15}$. Beside their functional roles in the pathogenesis of all known types of glomerular diseases, miR deregulations can play an important role as biomarkers ${ }^{16,17}$.

Currently the gold standard in miR quantification is RT- $q P_{C R}{ }^{18}$. For comprehensive analysis of RT-qPCR expression data, a reliable normalization strategy is required. The most widely applied method is the use of at least one endogenous reference gene $e^{19,20}$. The requirements for a suitable normalizer are sample type-independent abundant expression and a high stability throughout a certain sample set and it should not exhibit expressional differences between specific sub- and treatment groups.

Unfortunately, there is no consensus about a normalization strategy in zebrafish miR expression data. This is even more obvious when it comes to kidney research: Most studies make use of endogenous reference genes typically used in human and rodent studies. To the best of our knowledge there is no study specifically dealing with suitable endogenous normalizers focusing on zebrafish development and kidney disease models.

To address this, we analyzed the suitability of nine, from miR-sequencing preselected, miRs as endogenous reference genes for RT-qPCR based zebrafish miR expression data in different zebrafish strains, developmental stages and four different glomerular disease models.

\section{Materials and methods}

Zebrafish breeding. All zebrafish experiments have been performed in accordance with German law and local regulations approved and overseen by the "Landesamt für Landwirtschaft, Lebensmittelsicherheit und Fischerei, Rostock (LALLF)" of the federal state Mecklenburg-Western Pomerania. Embryonic zebrafish experiments (Morpholino-injections) until $5 \mathrm{dpf}$ do not require specialized ethics committee approval. Experiments beyond $5 \mathrm{dpf}$ were performed under the LALLF ethics approval \#7221.3-1-064/15. All experiments were performed in agreement with the ARRIVE guidelines. Zebrafish embryos were staged as described before ${ }^{21}$. Embryos were produced from timed matings as described ${ }^{22}$ and reared in E3 medium at $28.5^{\circ} \mathrm{C}$ in the dark with at least daily medium changes. Embryos of the following genotypes were used: AB/TÜ wildtype (ZDBGENO-010924-10), Casper (mitfa $\left.{ }^{\mathrm{w} 2 / \mathrm{w} 2}, \mathrm{mpv17}^{\mathrm{a} / \mathrm{a} 9}, \mathrm{ZDB}-\mathrm{FISH}-150901-6638\right), \mathrm{Tg}(\mathrm{fabp} 10 \mathrm{a}: \mathrm{gc}-\mathrm{eGFP}), \mathrm{mitfa}^{\mathrm{w} 2 / \mathrm{w} 2}$, mpv17 ${ }^{\text {a9/a9 }}$ (ZDB-FISH-160923-6) derived from double homozygous incrosses and Cherry (Tg(nphs2:GAL4); $\operatorname{Tg}(U A S$ :Eco.nfsB-mCherry) (mi1004Tg; rw0144Tg, ZDB-FISH-160601-223)). We used larvae of the three different strains at time points $24,48,72,96,120,144$ and $192 \mathrm{~h}$ past fertilization (hpf).

Drug treatment. Embryos with a $\operatorname{Tg}(n p h s 2: G A L 4), \operatorname{Tg}(U A S$ :Eco.nfsB-mCherry) background obtained from double transgenic incrosses and selected for strong and homogenous mCherry fluorescence in podocytes at $72 \mathrm{~h}$ past fertilization (hpf), were podocyte-depleted by treating embryos with $5 \mathrm{mM}$ metronidazole (MTZ) dissolved in 0.1\% DMSO in E3 medium or 0.1\% DMSO in E3 as a vehicle control from 96 to $120 \mathrm{hpf}$ as described before ${ }^{11}$. To induce focal and segmental glomerulosclerosis (FSGS)-like disease in zebrafish embryos, partial podocyte depletion was performed as described before ${ }^{12}$. Briefly, double transgenic $\operatorname{Tg}(n p h s 2:$ GAL4), $\operatorname{Tg}($ UAS:Eco.nfsB-mCherry) embryos were treated with $80 \mu \mathrm{M} \mathrm{MTZ}$ in $0.1 \%$ DMSO in E3 for $48 \mathrm{~h}$ starting at $96 \mathrm{hpf}$. For each treatment three independent biological replicates from individual clutches of embryos were set up.

Morpholino Injections. Antisense morpholino oligonucleotides were produced by Gene-Tools (Philomath, OR, USA). Following beforehand established and published morpholino sequences were used (MO IDs http://www.zfin.org): MO3-wtla (ZDB-MRPHLNO-071107-2), 5'-CACGAACATCAGAACCCATTTTGA G-3's; MO1-nphs1 (ZDB-MRPHLNO-051102-1), 5'-CGCTGTCCATTACCTTTAGGCTCC-3'9. Standard control morpholino targeting an intronic region of the human HBB gene: $5^{\prime}$ - CCTCTTACCTCAGTTACAATT TATA-3'. Lyophilized morpholinos were reconstituted in ultrapure water to a stock concentration of $1 \mathrm{mM}$. Before injections, target and control morpholinos were diluted to $100 \mu \mathrm{M}$ in injection solution containing $100 \mathrm{mM} \mathrm{KCl}, 10 \mathrm{mM}$ HEPES $\mathrm{pH} 7.6$ and $1 \%$ phenol red as a visual marker and incubated at $65{ }^{\circ} \mathrm{C}$ for 5 min to dissolve precipitates. Per embryo, $2 \mathrm{nl}$ were injected in the yolk of 1-2 cell-stage embryos. Injected embryos were collected in E3 and transferred to $10 \mathrm{~cm}$ petri dishes with fresh E3. Embryos were checked daily for viability and medium was changed twice a day. For each target, three independent clutches of embryos were injected.

Proteinuria assay. As described above, 1-cell embryos (Tg(fabp10a:gc-eGFP), mitfa ${ }^{\mathrm{w2} / \mathrm{w} 2}$, mpv17 $\left.{ }^{\mathrm{a} 9 / \mathrm{a} 9)}\right)$ expressing the $78 \mathrm{kDa}$ gc-eGFP fusion protein in the blood plasma were injected with respective morpholino oligonucleotides. Under healthy conditions, this fusion protein is retained in the vasculature. In the case of glomerular leakage, intravascular protein is filtered and lost with the urine. At $96 \mathrm{hpf}$, randomly selected larvae were imaged with a P2-SHR Plan Apo $1 \times$ objective attached to a SMZ18 fluorescence stereomicroscope equipped with a motorized Z-drive (Nikon GMBH, Düsseldorf, Germany) and an X-Cite Xylis LED (Excelitas, Göttingen, Germany) under exact the same illumination conditions for every group.

Sample generation. At the described endpoints, 20 embryos per group were collected in TRIreagent (Sigma Aldrich), homogenized using a tissue disruptor (MP-FastPrep-24, MPBiomedicals) with ceramic beads, snap-frozen in liquid nitrogen and stored at $-80^{\circ} \mathrm{C}$ upon RNA isolation. Total RNA isolation was performed as described per manufacturer's description. The RNA pellet was eluted in DEPC treated ultrapure water and stored at $-80^{\circ} \mathrm{C}$ upon further processing. RNA concentration and purity as 260/280 $\mathrm{nm}$ ratio was determined fluorometrically using an Eppendorf Biophotometer (Eppendorf, Hamburg, Germany). 
Candidate selection. Endogenous normalization candidates were selected from pretrials. Herein, smallRNA sequencing was performed from isolated glomeruli of zebrafish larvae (Tg(nphs2:GAL4); $\operatorname{Tg}($ UAS:Eco. nfsb-mCherry), ZFIN: ZDB-FISH-160,601-2 backcrossed to mitfa ${ }^{\text {w2/w2 }}$ ) treated with MTZ and DMSO as described above ${ }^{12}$ with slight changes. Glomeruli were isolated at $6 \mathrm{dpf}$ manually by micropipetting after slightly disrupting embryos in a tissue homogenizer. From the sequencing results we excluded miRNAs with less than 10 reads and p-values below 0.05 of pairwise comparisons between the treatment groups. The residual miRNAs were sorted by $\log _{2}$ fold changes between the two treatment groups. From these values we selected the most stable miRNAs as presented by values below 0.1 or above -0.1 . This resulted in the following 9 candidates: dre-miR-92a-3p, dre-miR-206-3p, dre-miR-99-1, dre-miR-92b-3p, dre-miR-363-3p, dre-let-7e, dre-miR-454a, dre-miR-30c-5p, dre-miR-126a-5p.

RT-PCR. For RT-PCR, $1 \mu \mathrm{g}$ of RNA was reverse-transcribed to cDNA using the Quantitect Reverse Transcription Kit (Qiagen) according to manufacturer's protocol. RT-PCR to assess MO knockdown efficiency was performed using the Phire Hot Start II DNA polymerase (Thermo Fisher Scientific) according to manufacturer's instructions with $1 \mu \mathrm{l}$ undiluted cDNA-template plus $19 \mu \mathrm{l}$ master mix containing primers targeting exon 24-26 of $n p h s 1$ and eflall1 as a reference gene. Primer sequences were: nphs1_exon24_F: GTCTATGTGGTGGTG ATCCTG, nphs1_exon26_R: CTGTGCCGAGGCGTTGATAA, efla111_F: AAGGAGGGTAATGCTAGCGG, ef1a1l1_R: GGGCGAAGGTCACAACCATA. As control a -RT, no template control from reverse-transcription and no template control from PCR Endpoint RT-PCR was run together with target samples in a Mastercycler gradient Thermocycler (Eppendorf) under following conditions: initial denaturation $98{ }^{\circ} \mathrm{C} 3 \mathrm{~min}, 33$ cycles: $98{ }^{\circ} \mathrm{C} 10 \mathrm{~s}, 60^{\circ} \mathrm{C} 20 \mathrm{~s}, 72{ }^{\circ} \mathrm{C} 25 \mathrm{~s}$. RT-PCR products were resolved on $3.5 \%$ low melting agarose (Biozym) in $1 \times$ TBE-buffer containing $0.16 \mu \mathrm{g} / \mathrm{ml}$ ethidium bromide.

Taqman miRNA assay. Starting with $10 \mathrm{ng}$ of total RNA, reverse transcription (RT) was performed using the Taqman miRNA Reverse Transcription Kit and Taqman miRNA Assays (Thermo Fisher Scientific). The following Taqman miRNA Assays were used: dre-miR-92a-3p Assay ID \#000431; dre-miR-206-3p Assay ID \#000501; dre-miR-99-1 Assay ID \#000435; dre-miR-92b-3p Assay ID \#007028_mat; dre-miR-363-3p Assay ID \#001271; dre-let-7e Assay ID \#005860_mat; dre-miR-454a Assay ID \#007306_mat; dre-miR-30c-5p Assay ID \#000419; dre-miR-126a-5p Assay ID \#000451. The RT reaction was performed after manufacturer's instruction using pooled primers. We included no template- as well as no reverse transcriptase controls. Additionally, a pooled RNA sample was used as positive control and inter-run calibrator that was synthesized with every RT run. RT-qPCR was performed using the above mentioned Taqman miRNA Assays and Taqman Universal Master Mix II, no UNG (Thermo Fisher Scientific) after manufacturer's instructions. All samples were run in triplicate and each reaction consisted of $1.33 \mu \mathrm{L}$ undiluted cDNA plus $18.7 \mu \mathrm{L}$ master mix. An additional no template control was added to the negative controls from the RT reaction. The qPCR was performed on the Bio-Rad iCycler Thermal Cycler with the iQ5 Multicolor Real-Time PCR Detection System (Bio-Rad, Hercules, CA, USA) with the following cycler scheme: $10 \mathrm{~min}$ at $95^{\circ} \mathrm{C}$ initial denaturation; 45 cycles of $15 \mathrm{~s}$ at $95^{\circ} \mathrm{C}$ and $60 \mathrm{~s}$ at $60^{\circ} \mathrm{C}$. The qPCR primary data analysis was done by the Bio-Rad iQ5 2.1 software with automatically set thresholds and baselines. Raw Ct-values $\geq 38$ were excluded from analysis. All Ct-values were inter-run-calibrator corrected.

Normalization analysis. The inter-run calibrator corrected values were analyzed for their stability by the online-based tool RefFinder ${ }^{24}$ (https://www.heartcure.com.au/reffinder/). It comprises the normalization tools BestKeeper ${ }^{25}$, comparative DeltaCt ${ }^{26}$, NormFinder ${ }^{27}$ and $\mathrm{GeNorm}^{28}$. These tools are based on different algorithms to evaluate the most stably expressed gene or gene pair of a specific sample set. For stability analysis we used different data sets: all values together, strains only, MTZ only and morpholinos only.

Statistical analysis. Statistical analysis was performed with IBM SPSS 22.0 (SPSS Inc., Chicago, IL, USA, https://www.ibm.com/de-de/analytics/spss-statistics-software) and GraphPad prism V5.01 (GraphPad Software, CA, USA, https://www.graphpad.com). Data was checked for gaussian distribution by Kolmogorov-Smirnov test. All groups were tested for statistically significant differences by two-way ANOVA and Bonferroni post-hoc test. All values are displayed as means with standard deviations. P-values $\leq 0.05$ were considered as statistically significant.

\section{Results}

Zebrafish kidney disease models: morpholino-mediated gene knockdown. As established and widely used kidney disease models, we injected 1-cell-stage embryos with antisense morpholino oligonucleotides targeting wt1a translation initiation (translation blocking MOs: TBM) and $n p h s 1$ splicing of the intron between $n p h s 1$ exons 24-25 (splice blocking MOs: SBM). While the transcription factor wt 1a plays an important role during glomerular development, $n p h s 1$ is a main component of the mature glomerular filtration barrier. Generally, as shown in Fig. 1A, injection of both MOs in 1-cell stage of zebrafish embryos resulted in typical pericardial edematous alterations ${ }^{8,9}$. Edema was graded in four categories from 0 to normal phenotype to 3 to severe whole-body edema with bent body axis. As shown in Fig. 1B,C, a statistically significant proportion of embryos injected with $2 \mathrm{nl}$ of $100 \mu \mathrm{M}$ anti-wt1a or anti- $n p h s 1$ morpholinos developed statistically significant pericardial or periocular edema. While the proportion of embryos showing edema of any severity was similar in both groups, the phenotype of the $n p h s 1$ knockdown embryos was generally more severe compared to the $w t 1 a$ knockdown (Fig. 1B). To investigate whether $w t 1 a$ and $n p h s 1$ knockdown induced high-molecular weight proteinuria, we injected 1-cell stage embryos expressing a $78 \mathrm{kDa}$ gc-eGFP fusion protein under control of the hepatocyte-specific fabp10a promotor. When produced, gc-eGFP is secreted in the blood plasma where it circu- 


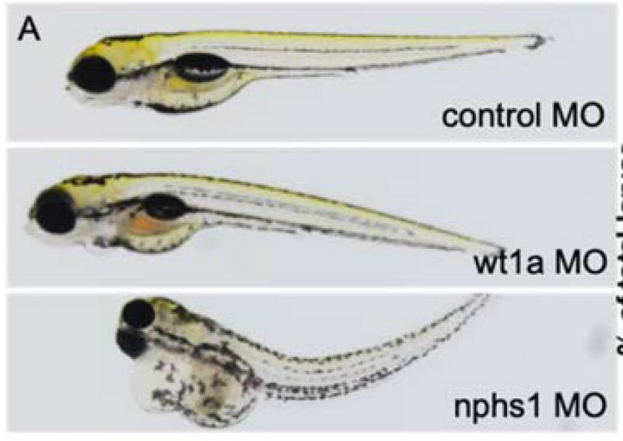

D

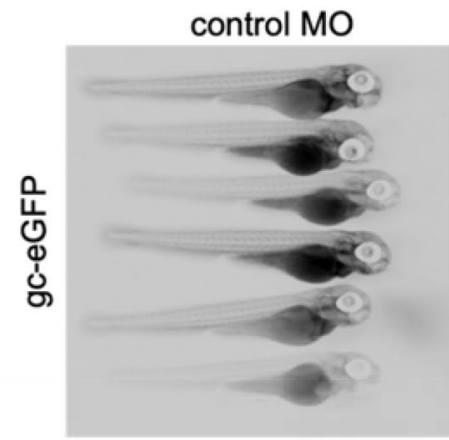

E

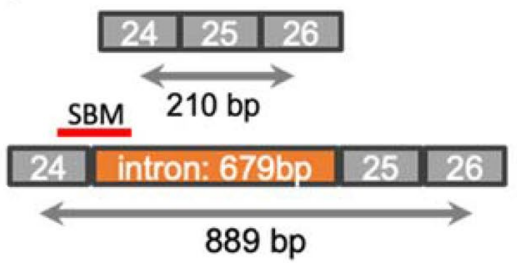

B

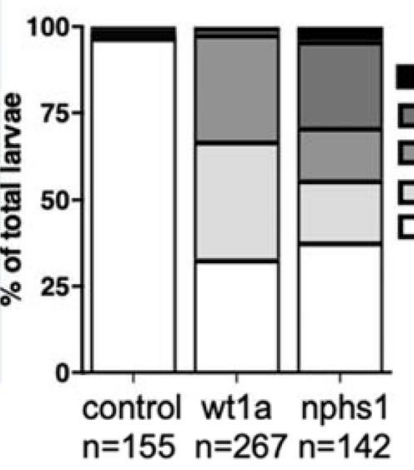

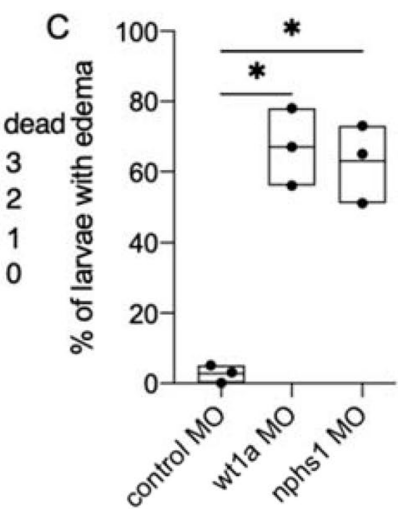

nphs1 MO
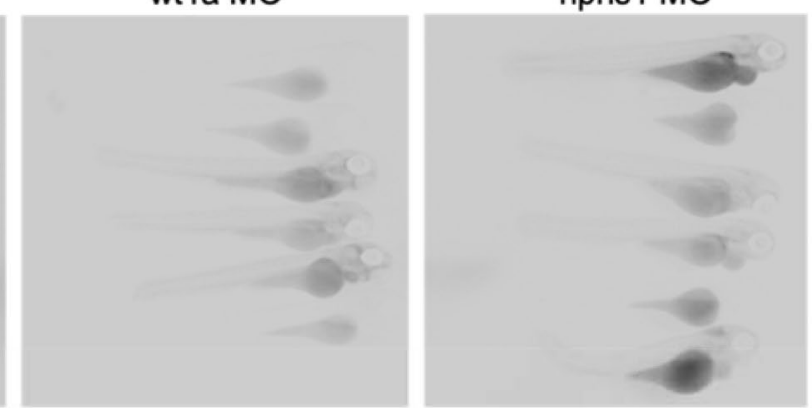

F control MO nphs1 MO -RT NTC $\mathrm{H}_{2} \mathrm{O}$

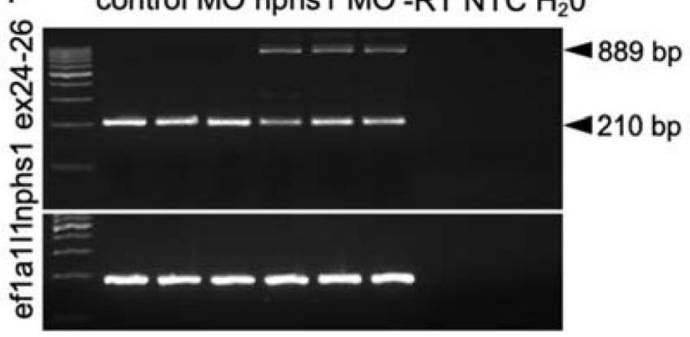

Figure 1. Induction of primary glomerular disease in larval zebrafish: Zebrafish embryos were injected with $2 \mathrm{nl} 100 \mu \mathrm{M}$ translation-blocking anti wtla, splice-blocking anti-nphs1 or non-binding control MOs. (A) Total of $n=564$ zebrafish embryos were injected and phenotyped at $120 \mathrm{hpf}$. Phenotype distribution graded in 0:no edema to 3: severe edema with bent body axis and dead larvae. As shown in the stacked boxplots in (B), the phenotype of the wtla knockdown larvae was generally more severe compared to nphs 1 knockdown while the general proportion of larvae with edema was similar in both groups $(\mathbf{C})$. No overt edema was seen in control $\mathrm{MO}$ injected larvae and the percentage of larvae with edema of any kind was statistically significant higher compared to paired control groups (paired t-test, $\mathrm{p}=0.01$ ). Shown in panel (D) are the results of the zebrafish proteinuria assay. While control MO-injected Tg(fabp10a:gc-eGFP) larvae showed intravascular localization of gc-eGFP, wt1a and nphs1 MO-injected larvae showed significantly less eGFP-fluorescence indicating clearance of the $78 \mathrm{kDa}$ fusion protein from the blood plasma. (E) shows the exon-intron structure of exons 24-26 in the nphs 1 gene with respective PCR-primer sites and the SBM MO binding site. Binding of the nphs1 SBM should lead to integration of a $679 \mathrm{bp}$ intron. (F) shows agarose gel-resolved RT-PCR products for the nphs1 region described in (D) for three independent control and nphs1 SBM injected groups. Arrowheads show the size of the respective wildtype-bands at 210 and the shifted PCR product after intronic integration.

lates and is retained under healthy conditions. Upon glomerular injury and impairment of the glomerular filtration barrier, this fusion protein will be rapidly cleared from the vasculature resulting in a decrease of vascular eGFP fluorescence ${ }^{34}$. As shown in Fig. 1D, at $96 \mathrm{hpf}$, control MO-injected embryos showed eGFP-fluorescence in the vasculature while $w t 1 a$ or $n p h s 1 \mathrm{MO}$-injected larvae showed significantly lower eGFP fluorescence indicative for leakage of protein (Fig. 1D, images with correlative bright field micrographs are shown in Suppl. Figure 1). As shown in the exon-intron scheme in Fig. 1E, the SBM used for the knockdown of $n p h s 1$ blocks the splice donor site leading to a truncated protein due to integration of the intron between exons 24 and 25. RT-PCR (oligo positions depicted in Fig. 1E) amplifying exons 24-26 showed integration of the intron only after injection of the $n p h s 1$ SBM (889 bp) with down-regulation of the wild type allele (210 bp) not containing the intron. As a reference gene efla1l1 was used which was stably expressed across samples (Fig. 1F). 


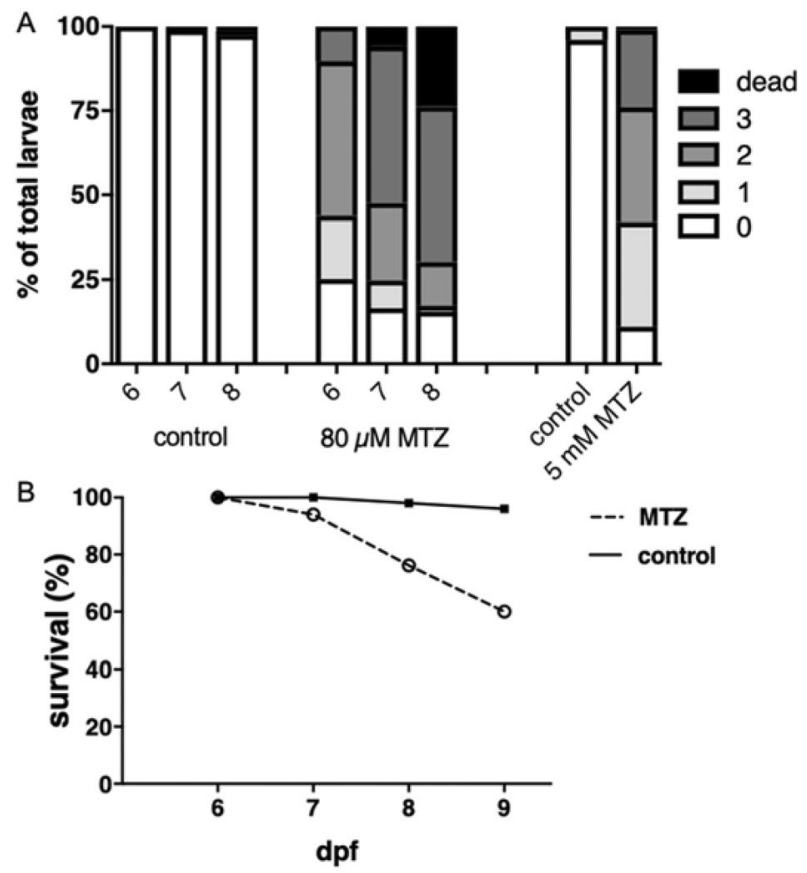

Figure 2. Acute and chronic zebrafish podocyte depletion models: (A) Two models of podocyte depletion were used: First, $80 \mu \mathrm{M}$ MTZ was applied over $48 \mathrm{~h}$. Edema developed in the proportions shown in (A). As shown in (B), mortality increased significantly $48 \mathrm{~h}$ after MTZ washout.

Zebrafish kidney disease models: pharmacogenetic podocyte depletion. Two protocols to injury adult podocytes were used: First, embryos expressing bacterial nitroreductase (NTR) specifically in podocytes were treated with a high concentration of MTZ $(5 \mathrm{mM})$ from 4 to $5 \mathrm{dpf}$ to deplete most podocytes from the GBM. This induces an acute onset of proteinuria mimicking acute nephrotic syndrome. As shown in the graph in Fig. 2A, 89\% of larvae developed significant edema after $24 \mathrm{~h}$ of treatment with $5 \mathrm{mM}$ MTZ while only $4 \%$ of $0.1 \%$ DMSO control-treated larvae developed edema. This model leads to a rather acute form of podocyte injury and induces a rapid-onset nephrotic syndrome in zebrafish larvae.

In contrast to that, a lower concentration of MTZ depleted only a subset of podocytes and leads to a prolonged disease course. This mimics human FSGS with its diverse features (progressive proteinuria, parietal epithelial cell activation, extracellular matrix deposition) as we have shown before ${ }^{12}$. As shown in Fig. $2 \mathrm{~A}$, the phenotype progressively developed during the disease course over 4 days after the washout of MTZ with a lower concentration with increasing lethality until $9 \mathrm{dpf}$ (Fig. 2B).

Expression of candidate as endogenous controls. We investigated the expression of dre-miR-92a-3p, dre-miR-206-3p, dre-miR-99-1, dre-miR-92b-3p, dre-miR-363-3p, dre-let-7e, dre-miR-454a, dre-miR-30c-5p and dre-miR-126a-5p by RT-qPCR. Mean Ct-values of the single samples were inter-run calibrator corrected. We observed an abundant expression of all candidate miRs in all samples. All candidate miRs showed a relatively homogenous expression pattern throughout the different strains and treatment groups (Fig. 3). Dre-miR-92b-3p showed the lowest standard deviation (SD) of 0.018 followed by dre-miR-92a-3p (SD=0.23), dre-miR-206-3p $(\mathrm{SD}=0.029)$, dre-miR-363-3p ( $\mathrm{SD}=0.030)$, dre-miR-99-1 $(\mathrm{SD}=0.032)$, dre-miR-30c-5p $(\mathrm{SD}=0.032)$, dre-miR$126 \mathrm{a}-5 \mathrm{p}(\mathrm{SD}=0.035)$, dre-miR-454a $(\mathrm{SD}=0.036)$ and dre-miR-let7e $(\mathrm{SD}=0.047)$, respectively.

Stability analysis. The IRC corrected miRNA expression data was analyzed by the combined online tool RefFinder. For the analysis we used all unsorted values together as well as subclassifications such as only strains, only MTZ treatments and only morpholino-mediated knockdowns as input. The data was analyzed by BestKeeper, Genorm, Normfinder and DeltaCt.

BestKeeper. BestKeeper ranked the normalization candidates within the unsorted values from most stable to less stable as follows: dre-miR-92b-3p (0.015), dre-miR-92a-3p (0.017), dre-miR-206-3p (0.021), dre-miR363-3p (0.021), dre-miR-99-1 (0.022), dre-miR-30c-5p (0.025), dre-miR-126a-5p (0.026), dre-miR-454a (0.028), dre-let-7e (0.034). dre-miR-126a-5p (0.023), dre-miR-30c-5p (0.03), dre-miR-99-1 (0.032), dre-miR-454a (0.033), dre-let-7e (0.044).

Looking at strains only, BestKeeper ranked the candidate miRs in the following order: dre-miR-92b-3p (0.016), dre-miR-92a-3p (0.020), dre-miR-363-3p (0.022), dre-miR-206-3p (0.023), dre-miR-126a-5p (0.023), dre-miR-30c-5p (0.030), dre-miR-99-1 (0.032), dre-miR-454a (0.033), dre-let-7e (0.044). 

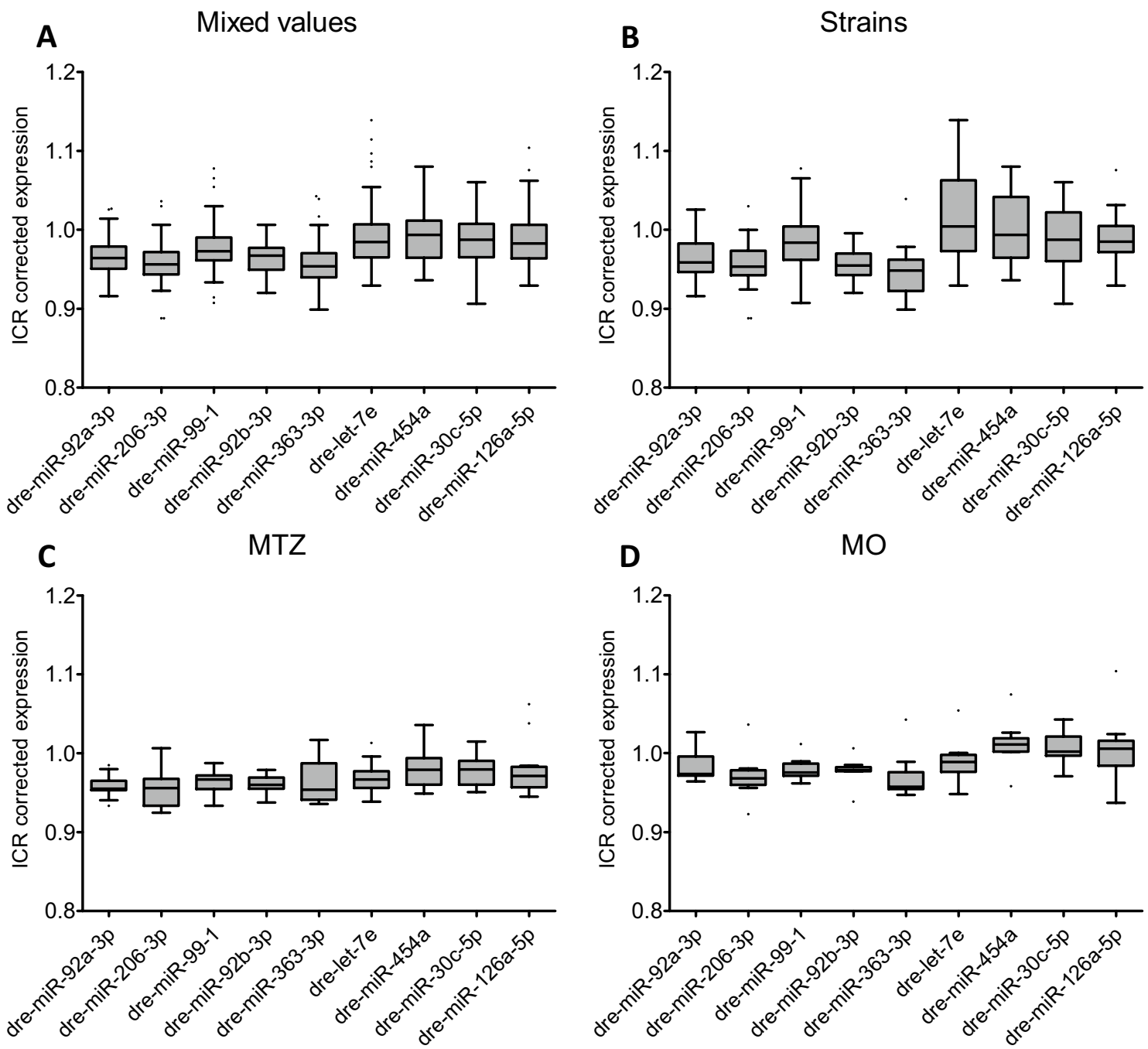

Figure 3. Abundant expression of candidate as endogenous control miRs. All miR candidates were detectable in the whole sample set. In general, they exhibit a high homogeneity as indicated by low standard deviations (SD). MiR-92b-3p shows most homogenous expression levels in mixed values (A), strains only (B), MTZtreatment group (C) and in MO-treated group (D). Data is presented as inter-run calibrator (ICR) corrected expression. Box-Whisker plots with upper and lower quartiles and outliers from technical triplicates. (A) 45 samplings of 20 pooled larvae; (B) 21 samplings of 20 pooled larvae; (C) 15 samplings of 20 pooled larvae; (D) 9 samplings of 20 pooled larvae.

Based on the expression data obtained from MTZ treatments the ranking changed in the following way: dremiR-92b-3p (0.009), dre-miR-92a-3p (0.011), dre-miR-99-1 (0.011), dre-let-7e (0.015), dre-miR-206-3p (0.016), dre-miR-30c-5p (0.016), dre-miR-454a (0.018), dre-miR-126a-5p (0.022), dre-miR-363-3p (0.023).

The RT-qPCR results from the morpholino-knockdown subset resulted in the following ranking: dre-miR92b-3p (0.009), dre-miR-99-1 (0.010), dre-miR-30c-5p (0.015), dre-miR-92a-3p (0.016), dre-miR-454a (0.017), dre-let-7e (0.017), dre-miR-206-3p (0.018), dre-miR-363-3p (0.021), dre-miR-126a-5p (0.027) (Fig. 4).

Genorm. The second normalization determination software Genorm ranked the miR candidates based on the mixed values as follows: dre-miR-454a (0.012), dre-miR-30c-5p (0.012), dre-miR-99-1 (0.015), dre-miR-206-3p (0.018), dre-miR-92b-3p (0.021), dre-miR-92a-3p (0.022), dre-miR-126a-5p (0.024), dre-miR-363-3p (0.026), dre-let-7e (0.028).

The stability ranking within the strains only showed this order: dre-miR-92a-3p (0.008), dre-miR-92b-3p (0.008), dre-miR-206-3p (0.014), dre-miR-126a-5p (0.015), dre-miR-30c-5p (0.02), dre-miR-454a (0.021), dremiR-99-1 (0.023), dre-miR-363-3p (0.025), dre-let-7e (0.028).

Genorm ranked the miR candidates based on MTZ treatment expression values like this: dre-miR-92a-3p (0.007), dre-miR-92b-3p (0.007), dre-miR-99-1 (0.009), dre-let-7e (0.01), dre-miR-30c-5p (0.01), dre-miR-454a (0.011), dre-miR-206-3p (0.012), dre-miR-363-3p (0.015), dre-miR-126a-5p (0.018).

The potential endogenous controls within the morpholino subset were ranked as following: dre-miR-206-3p (0.005), dre-let-7e (0.005), dre-miR-454a (0.006), dre-miR-30c-5p (0.009), dre-miR-92b-3p (0.011), dre-miR-99-1 (0.012), dre-miR-363-3p (0.016), dre-miR-92a-3p (0.02), dre-miR-126a-5p (0.025) (Fig. 4). 

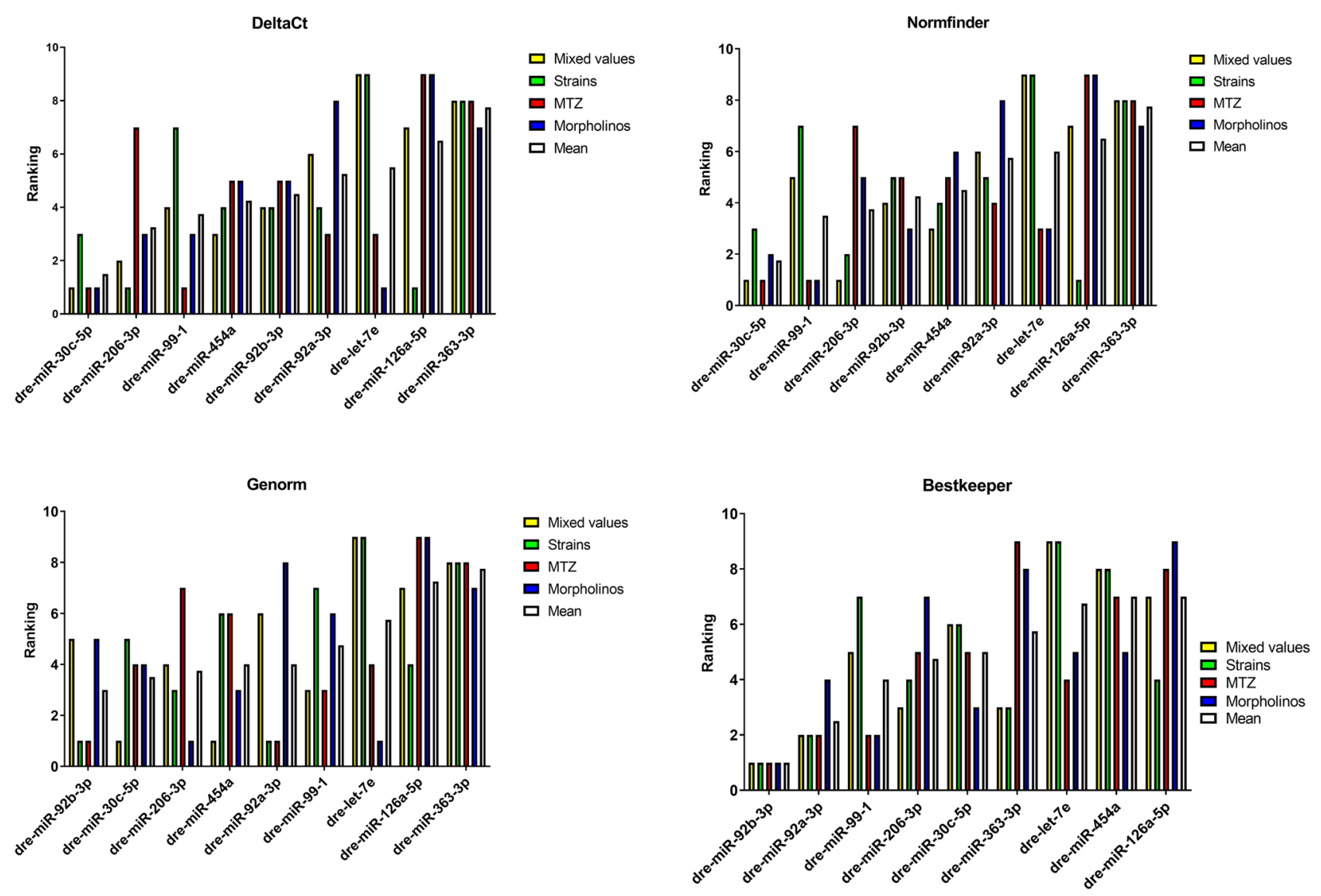

Figure 4. Ranking of potential endogenous control miRs by 4 different normalization determination softwares. Data was ranked by DeltaCt, Normfinder, Genorm and Bestkeeper with respect to mixed values, strains only, MTZ only and morpholinos only as well as the mean rank.

Normfinder. With respect to the mixed values Normfinder software shows this ranking: dre-miR-206-3p (0.011), dre-miR-30c-5p (0.011), dre-miR-454a (0.014), dre-miR-92b-3p (0.016), dre-miR-99-1 (0.017), dremiR-92a-3p (0.019), dre-miR-126a-5p (0.025), dre-miR-363-3p (0.03), dre-let-7e (0.032).

Normfinder ranked the potential normalizers with expression values from strains only as follows: dre-miR126a-5p (0.007), dre-miR-206-3p (0.011), dre-miR-30c-5p (0.016), dre-miR-454a (0.017), dre-miR-92b-3p (0.019), dre-miR-92a-3p (0.019), dre-miR-99-1 (0.021), dre-miR-363-3p (0.032), dre-let-7e (0.035).

The MTZ-treatments resulted in the following order: dre-miR-99-1 (0.006), dre-miR-30c-5p (0.006), drelet-7e (0.007), dre-miR-92a-3p (0.009), dre-miR-454a (0.01), dre-miR-92b-3p (0.01), dre-miR-206-3p (0.012), dre-miR-363-3p (0.02), dre-miR-126a-5p (0.029).

The ranking of the morpholino treatment group showed the following order: dre-miR-99-1 (0.005), dremiR-30c-5p (0.007), dre-miR-92b-3p (0.008), dre-let-7e (0.008), dre-miR-206-3p (0.01), dre-miR-454a (0.012), dre-miR-363-3p (0.025), dre-miR-92a-3p (0.029), dre-miR-126a-5p (0.043) (Fig. 4).

DeltaCt. Delta Ct ranked the potential endogenous control miRs from mixed values as shown in the following: dre-miR-30c-5p (0.023), dre-miR-206-3p (0.024), dre-miR-454a (0.025), dre-miR-92b-3p (0.026), dre-miR-99-1 (0.026), dre-miR-92a-3p (0.027), dre-miR-126a-5p (0.032), dre-miR-363-3p (0.034), dre-let-7e (0.036).

When looking at the three different strains only the ranking was the following: dre-miR-126a-5p (0.023), dremiR-206-3p (0.023), dre-miR-30c-5p (0.025), dre-miR-454a (0.026), dre-miR-92a-3p (0.026), dre-miR-92b-3p (0.026), dre-miR-99-1 (0.028), dre-miR-363-3p (0.035), dre-let-7e (0.038).

DeltaCt ranked the miR candidates based on MTZ-treatment values as follows: dre-miR-99-1 (0.014), dremiR-30c-5p (0.014), dre-let-7e (0.015), dre-miR-92a-3p (0.015), dre-miR-92b-3p (0.016), dre-miR-454a (0.016), dre-miR-206-3p (0.018), dre-miR-363-3p (0.024), dre-miR-126a-5p (0.031).

The morpholino knockdown groups resulted in the following ranking: dre-let-7e (0.019), dre-miR-30c-5p (0.019), dre-miR-99-1 (0.02), dre-miR-206-3p (0.02), dre-miR-92b-3p (0.021), dre-miR-454a (0.021), dre-miR363-3p (0.03), dre-miR-92a-3p (0.033), dre-miR-126a-5p (0.045) (Fig. 4).

Average ranking. For determination of the most stable normalization candidate miR, we calculated the mean rank for every single candidate within the specific determination software as well as the summarized stability score (SSS) ${ }^{29}$. This revealed that dre-miR-206-3p is the most stable candidate miR throughout the different 


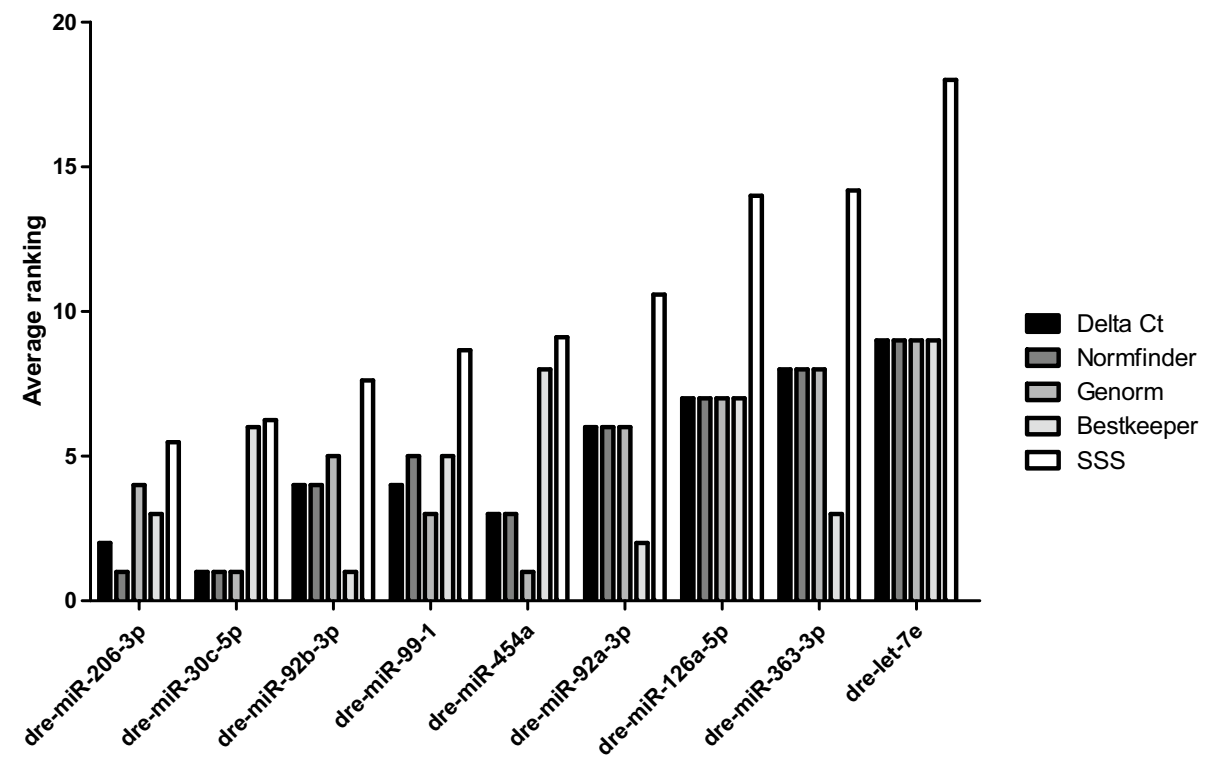

Figure 5. Average ranking of candidate miRs by 4 different normalization determination algorithms. Data was ranked by DeltaCt, Normfinder, Genorm and Bestkeeper. SSS = summarized stability score.

treatment and sample types which got the lowest SSS of 5.5. It was closely followed by dre-miR-30c-5p (6.2), dre-miR-92b-3p (7.6), dre-miR-99-1 (8.7), dre-miR-454a (9.1), dre-miR-92a-3p (10.6), dre-miR-126a-5p (14.0), dre-miR-363-3p (14.2), and dre-let-7e (18.0) as the least stable candidate miR (Fig. 5). Additionally, treatment subgroups have been ranked as described above. Results of this subgroup ranking is plotted in Suppl. Figure 2.

Inter-group differences. Since a certain endogenous control should not only be stable throughout a specific sample set but should not show significant expression differences between sample subgroups and treatment groups, we tested the expression data of the single miRs for the aforementioned differences. We could not find statistically significant expression differences between the three different strains for any tested miR. Additionally, there were no significant differences between the MTZ-treated groups themselves as well as between the treatment- and the control-groups. This was also true for the morpholino groups, where we could not detect any significant differences between the $n p h s 1$ - and $w t 1 a$ MOs groups as well as between them and the control group (Fig. 6).

Developmental differences. Beside specific developmental zebrafish research, many studies are dealing with zebrafish larvae at different developmental stages. In case of miR-based RT-qPCR data this fact sets the requirement for an endogenous control that shows either a high stability or no significant differences between developmental stages. To address this, we tested our data set for differences between the single developmental stages. For dre-miR-92a-3p, dre-miR-92b-3p and miR-126a-5p we could not detect any significant differences. The other six candidates showed differences between different developmental stages. Dre-miR-206-3p showed significant differences between 72 and $120 \mathrm{~h}$ and between 96 and $120 \mathrm{~h}$. The next candidate, dre-miR-99-1, showed significant differences between $24 \mathrm{~h}$ and the other time points. Dre-miR-363-3p had significant expression differences between 120 and $72 \mathrm{~h}$ and between 120 and $48 \mathrm{~h}$. Dre-miR-let7e showed differences between $24 \mathrm{~h}$ and all other time points as well as between 48 and $72 \mathrm{~h}, 96 \mathrm{~h}, 144 \mathrm{~h}$ and $192 \mathrm{~h}$, respectively. The next candidate dre-miR-454a exhibited differences between 24 and 72 h, 96 h, 144 h and 192 h, respectively, as well as between 48 and $96 \mathrm{~h}$. Interestingly, we could observe significant differences between developmental stages in the expression data of the most stably ranked normalization candidate dre-miR-30c-5p. It showed significant differences between 24 and 72 h, 96 h, 144 h and 192 h, respectively, and between 48 and 96 h (Fig. 7).

\section{Discussion}

Currently, there is no consensus about endogenous reference controls for RT-qPCR derived miR expression data in zebrafish larvae. To best of our knowledge, there are no studies explicitly approaching different drug treatments, knockdowns and/or developmental stages like it is the case for mRNAs ${ }^{30,31}$. In the present study we investigated the nine preselected candidates dre-miR-92a-3p, dre-miR-206-3p, dre-miR-99-1, dre-miR-92b-3p, dre-miR-363-3p, dre-let-7e, dre-miR-454a, dre-miR-30c-5p and dre-miR-126a-5p for their suitability as endogenous reference genes. As it has been shown before, zebrafish embryos exhibit highly dynamic miR expression patterns during development among different developmental stages in three different genetic zebrafish strains ${ }^{2,32}$. We started at $24 \mathrm{~h}$ past fertilization (hpf), because lower time points have only a little relevance for kidney research ${ }^{6}$ and many miRs show no or only little expression below this time point or are influenced by the 
dre-miR-let-7e

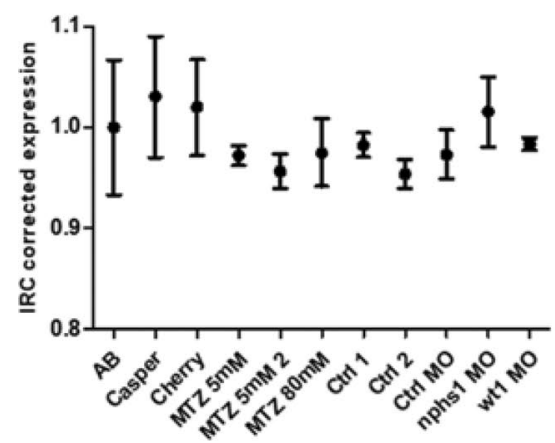

dre-miR-126a-5p

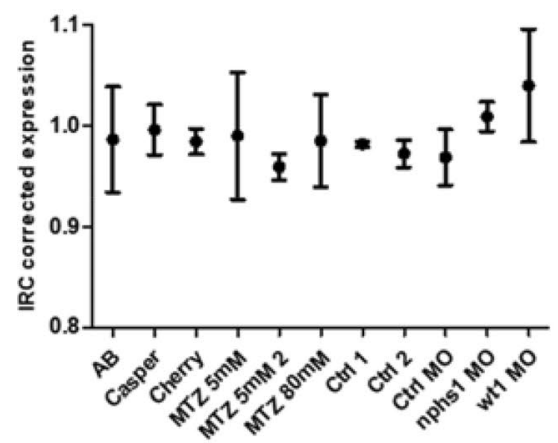

dre-miR-206-3p

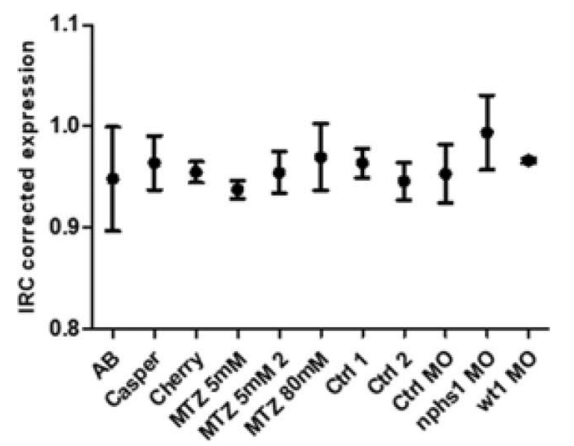

dre-miR-30c-5p

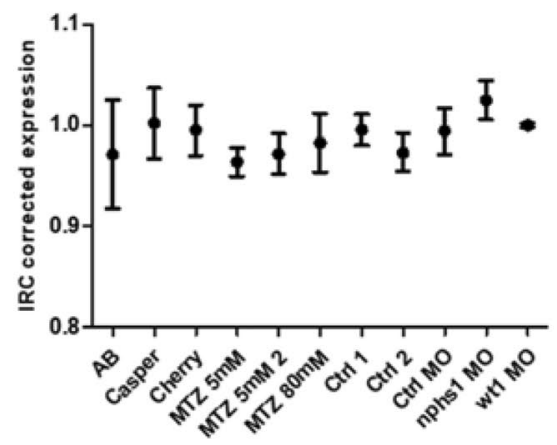

dre-miR-363-3p

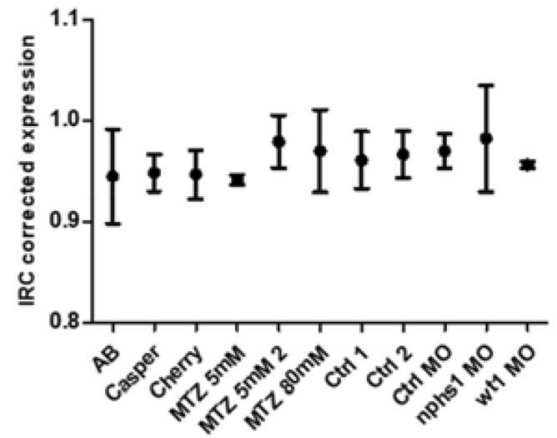

dre-miR-454a

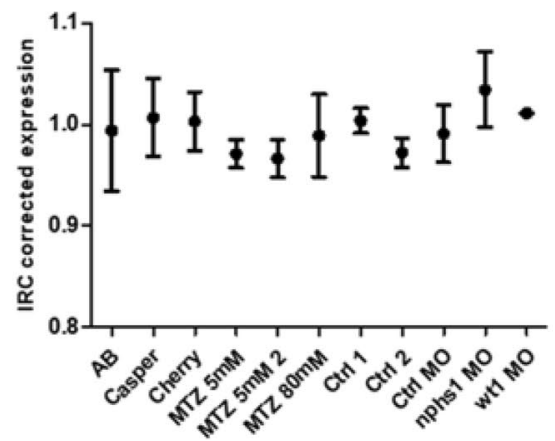

dre-miR-92a-5p

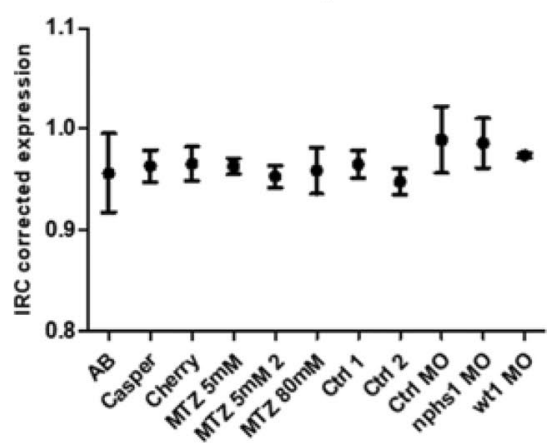

dre-miR-92b-3p

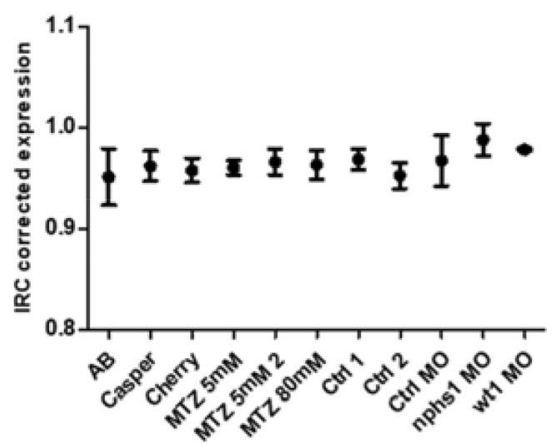

dre-miR-99-1

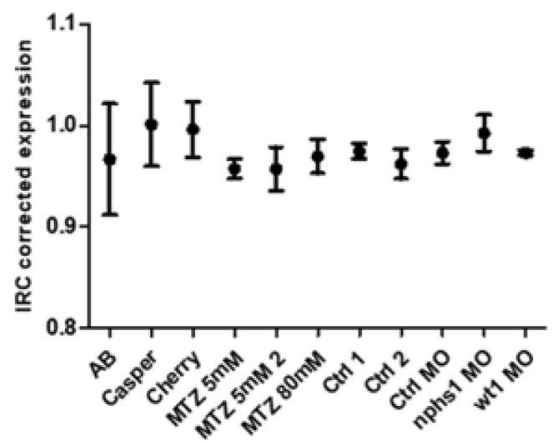

Figure 6. Differences in candidate miR expression between different subgroups. There are no significant differences between the presented subgroups. Data is presented as inter-run calibrator (ICR) corrected expression. All values are shown as mean from technical triplicates. Error bars $=$ SD.

abundance of maternal miRs in the yolk ${ }^{32}$. Additionally, to different background strains and the developmental series, we applied four different models, successfully established to model glomerular diseases in zebrafish:

The first two models are based on the morpholino-guided knockdown of genes important for glomerular development and/or maintenance of the glomerular filtration barrier. The first one hindered translation initiation of $w t 1 a$, a transcription factor that regulates proper glomerular development and is still active in adult podocytes. The second one targeted proper splicing of $n p h s 1$, a gene encoding the protein nephrin which is a crucial part of the slit diaphragm and therefore maintaining glomerular filtration barrier function. In line with our results, knockdown of both genes has been shown to result in disrupted glomerular development as well as in early onset of high molecular weight proteinuria in larval zebrafish ${ }^{8,9}$.

The other models represent a pharmacogenetic form of highly specific podocyte depletion. They are grounded on the NTR/MTZ model of targeted tissue ablation which has been translated to podocyte research. In this model expression of the NTR, a bacterial enzyme, is transgenically driven by tissue-specific promotor fragments, such as in our case the $n p h s 2$ promotor. When zebrafish embryos are treated with the antibiotic MTZ, the NTR converts this prodrug into a cytotoxin which leads to rapid apoptosis in the targeted tissue. Initially this model has been established to deplete pancreatic beta-cells and has later been modified to a dose-dependent depletion of podocytes ${ }^{10,33}$. We have shown that upon treatment with high dose MTZ (5 mM) zebrafish rapidly develop proteinuria and classic morphologic changes known from human nephrotic syndrome such as severe podocyte foot process effacement and later podocyte detachment ${ }^{11,34}$. Lately, we could show that when dose-dependently only a smaller subset of podocytes is depleted, a more chronic course of disease is initiated. In that model, zebrafish 


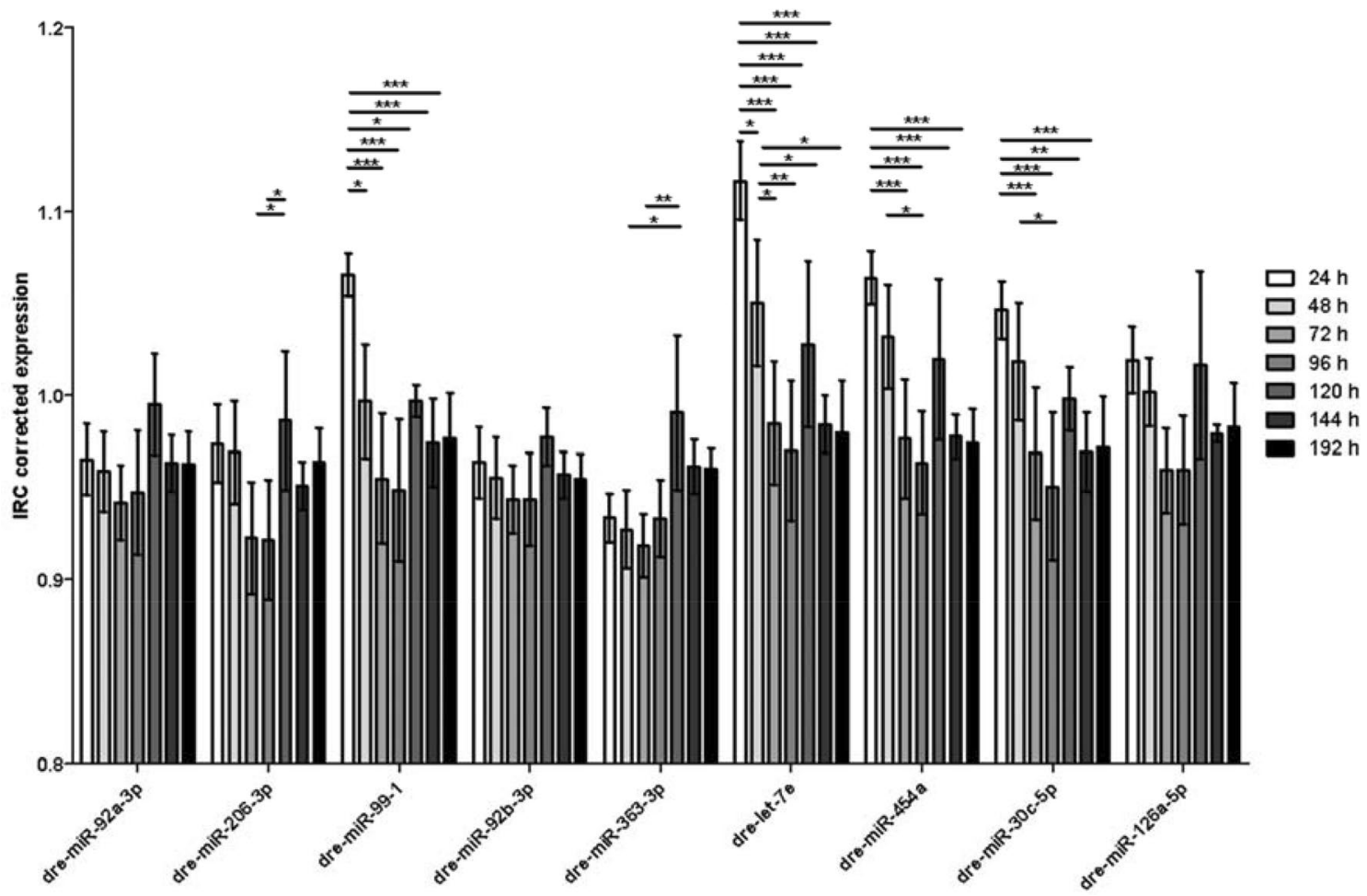

Figure 7. Differences in candidate miR expression between different developmental stages. There are no significant differences between the presented developmental stages in the expression of dre-miR-92a-3p, dremiR-92b-3p and dre-miR-126a-5p. Data is presented as inter-run calibrator (ICR) corrected expression. All values are shown as mean from technical triplicates. ${ }^{*} \mathrm{p} \leq 0.05,{ }^{* *} \mathrm{p} \leq 0.01,{ }^{* * *} \mathrm{p} \leq 0.001$. Error bars $=\mathrm{SD}$.

embryos resemble the phenotype known from human FSGS including activation of parietal epithelial cells that migrate to the glomerular tuft and deposit extracellular matrix ${ }^{12}$.

After RT-qPCR based measurements, the expression levels of candidate miRs showed a high homology in general in the described models. There were no statistically significant differences detectable between the single subgroups, except for developmental stages. The SSS revealed dre-miR-206-3p as the most stable endogenous reference gene. Dre-miR-206-3p has been shown to play a crucial role in angiogenesis of zebrafish larvae ${ }^{35}$. It was followed by dre-miR-30c-5p which is known as a key player in pronephric development in Xenopus laevis and Danio rerio, where it shows a pronephros specific expression within the first days of development ${ }^{36,37}$. It has also been used as an endogenous reference gene before ${ }^{38}$. Unfortunately, there were significant differences in the expression of dre-miR-206-3p and dre-miR-30c-5p between different developmental stages, so they had to be discarded as suitable normalizers for this type of experimental set-ups. The only miRs that showed no differences in their expression levels between developmental stages were dre-miR-92a-3p, dre-miR-92b-3p and dremiR-126a-5p. Dre-miR-92b-3p was the third most stable candidate closely after dre-miR-30c-5p. Additionally, it was exclusively ranked first in all subgroups by Bestkeeper. Dre-miR-92b-3p is known to be abundantly and ubiquitously expressed in zebrafish up from $24 \mathrm{hpf}^{39}$ and is essential, together with dre-miR-92a, for the earliest steps in zebrafish embryogenesis ${ }^{40}$.

The present study shows that all tested candidate miRs have a high homology within the tested experimental setups and that the most stable normalizer was dre-miR-206-3p. However, if it comes to developmental research studies or comparisons of zebrafish larvae at different ages it is rather unsuitable. Our analysis shows that dremiR-92b-3p is the best candidate to be used as an endogenous reference gene for RT-qPCR-based miR expression in zebrafish larval studies.

Received: 9 April 2021; Accepted: 6 September 2021

Published online: 24 November 2021

\section{References}

1. Bartel, D. P. MicroRNAs: Target recognition and regulatory functions. Cell 136(2), 215-233. https://doi.org/10.1016/j.cell.2009. 01.002 (2009).

2. Bhattacharya, M. et al. The crucial role and regulations of miRNAs in zebrafish development. Protoplasma 254(1), 17-31. https:// doi.org/10.1007/s00709-015-0931-1 (2017).

3. Muller Deile, J. \& Schiffer, M. The zebrafish model to study the role of microRNAs in glomerular function and disease. Adv. Tech. Biol. Med. https://doi.org/10.4172/2379-1764.1000230 (2017). 
4. Morales, E. E. \& Wingert, R. A. Zebrafish as a model of kidney disease. Results Probl. Cell Differ. 60, 55-75. https://doi.org/10. 1007/978-3-319-51436-9_3 (2017).

5. Rahman Khan, F. \& Sulaiman Alhewairini, S. Zebrafish (Danio rerio ) as a Model Organism. In Current Trends in Cancer Management (eds Streba, L. et al.) (IntechOpen, 2019).

6. Drummond, I. A. \& Davidson, A. J. Zebrafish kidney development. Methods Cell Biol. 100, 233-260. https://doi.org/10.1016/ B978-0-12-384892-5.00009-8 (2010).

7. Kriz, W. Podocyte is the major culprit accounting for the progression of chronic renal disease. Microsc. Res. Tech. 57(4), 189-195. https://doi.org/10.1002/jemt.10072 (2002).

8. Perner, B., Englert, C. \& Bollig, F. The Wilms tumor genes wtla and wt $1 \mathrm{~b}$ control different steps during formation of the zebrafish pronephros. Dev. Biol. 309(1), 87-96. https://doi.org/10.1016/j.ydbio.2007.06.022 (2007).

9. Kramer-Zucker, A. G., Wiessner, S., Jensen, A. M. \& Drummond, I. A. Organization of the pronephric filtration apparatus in zebrafish requires Nephrin, Podocin and the FERM domain protein Mosaic eyes. Dev. Biol. 285(2), 316-329. https://doi.org/10. 1016/j.ydbio.2005.06.038 (2005).

10. Zhou, W. \& Hildebrandt, F. Inducible podocyte injury and proteinuria in transgenic zebrafish. J. Am. Soc. Nephrol. 23(6), 10391047. https://doi.org/10.1681/ASN.2011080776 (2012).

11. Siegerist, F., Blumenthal, A., Zhou, W., Endlich, K. \& Endlich, N. Acute podocyte injury is not a stimulus for podocytes to migrate along the glomerular basement membrane in zebrafish larvae. Sci. Rep. 7, 43655. https://doi.org/10.1038/srep43655 (2017).

12. Hansen, K. U. I. et al. Prolonged podocyte depletion in larval zebrafish resembles mammalian focal and segmental glomerulosclerosis. FASEB J. 34(12), 15961-15974. https://doi.org/10.1096/f.202000724R (2020).

13. Harvey, S. J. et al. Podocyte-specific deletion of dicer alters cytoskeletal dynamics and causes glomerular disease. J. Am. Soc. Nephrol. 19(11), 2150-2158. https://doi.org/10.1681/ASN.2008020233 (2008).

14. Shi, S. et al. Podocyte-selective deletion of dicer induces proteinuria and glomerulosclerosis. J. Am. Soc. Nephrol. 19(11), 2159-2169. https://doi.org/10.1681/ASN.2008030312 (2008).

15. Zhdanova, O. et al. The inducible deletion of Drosha and microRNAs in mature podocytes results in a collapsing glomerulopathy. Kidney Int. 80(7), 719-730. https://doi.org/10.1038/ki.2011.122 (2011).

16. Connor, K. L. \& Denby, L. MicroRNAs as non-invasive biomarkers of renal disease. Nephrol. Dial. Transplant. https://doi.org/10. 1093/ndt/gfz183 (2019).

17. Ramanathan, K. \& Padmanabhan, G. MiRNAs as potential biomarker of kidney diseases: A review. Cell Biochem. Funct. 38(8), 990-1005. https://doi.org/10.1002/cbf.3555 (2020).

18. Schmittgen, T. D. et al. Real-time PCR quantification of precursor and mature microRNA. Methods 44(1), 31-38. https://doi.org/ 10.1016/j.ymeth.2007.09.006 (2008).

19. Schwarzenbach, H., Da Silva, A. M., Calin, G. \& Pantel, K. Data normalization strategies for microRNA quantification. Clin. Chem. 61(11), 1333-1342. https://doi.org/10.1373/clinchem.2015.239459 (2015).

20. Meyer, S. U., Pfaffl, M. W. \& Ulbrich, S. E. Normalization strategies for microRNA profiling experiments: A "normal" way to a hidden layer of complexity?. Biotechnol. Lett. 32(12), 1777-1788. https://doi.org/10.1007/s10529-010-0380-z (2010).

21. Kimmel, C. B., Ballard, W. W., Kimmel, S. R., Ullmann, B. \& Schilling, T. F. Stages of embryonic development of the zebrafish. Dev. Dyn. 203(3), 253-310. https://doi.org/10.1002/aja.1002030302 (1995).

22. Müller, T. et al. Non-muscle myosin IIA is required for the development of the zebrafish glomerulus. Kidney Int. 80(10), 1055-1063. https://doi.org/10.1038/ki.2011.256 (2011).

23. Chen, Z. et al. GADD45B mediates podocyte injury in zebrafish by activating the ROS-GADD45B-p38 pathway. Cell Death Dis. 7, e2068. https://doi.org/10.1038/cddis.2015.300 (2016).

24. Xie, F., Xiao, P., Chen, D., Xu, L. \& Zhang, B. miRDeepFinder: A miRNA analysis tool for deep sequencing of plant small RNAs. Plant Mol. Biol. https://doi.org/10.1007/s11103-012-9885-2 (2012).

25. Pfaffl, M. W., Tichopad, A., Prgomet, C. \& Neuvians, T. P. Determination of stable housekeeping genes, differentially regulated target genes and sample integrity: BestKeeper-Excel-based tool using pair-wise correlations. Biotechnol. Lett. 26(6), 509-515. https://doi.org/10.1023/b:bile.0000019559.84305.47 (2004).

26. Silver, N., Best, S., Jiang, J. \& Thein, S. L. Selection of housekeeping genes for gene expression studies in human reticulocytes using real-time PCR. BMC Mol Biol. 7, 33. https://doi.org/10.1186/1471-2199-7-33 (2006).

27. Andersen, C. L., Jensen, J. L. \& Ørntoft, T. F. Normalization of real-time quantitative reverse transcription-PCR data: A model-based variance estimation approach to identify genes suited for normalization, applied to bladder and colon cancer data sets. Cancer Res. 64(15), 5245-5250. https://doi.org/10.1158/0008-5472.CAN-04-0496 (2004).

28. Vandesompele, J. et al. Accurate normalization of real-time quantitative RT-PCR data by geometric averaging of multiple internal control genes. Genome Biol. https://doi.org/10.1186/gb-2002-3-7-research0034 (2002).

29. Marabita, F. et al. Normalization of circulating microRNA expression data obtained by quantitative real-time RT-PCR. Brief Bioinform. 17(2), 204-212. https://doi.org/10.1093/bib/bbv056 (2016).

30. McCurley, A. T. \& Callard, G. V. Characterization of housekeeping genes in zebrafish: Male-female differences and effects of tissue type, developmental stage and chemical treatment. BMC Mol. Biol. 9, 102. https://doi.org/10.1186/1471-2199-9-102 (2008).

31. Casadei, R. et al. Identification of housekeeping genes suitable for gene expression analysis in the zebrafish. Gene Expr. Patterns. 11(3-4), 271-276. https://doi.org/10.1016/j.gep.2011.01.003 (2011).

32. Chen, P. Y. et al. The developmental miRNA profiles of zebrafish as determined by small RNA cloning. Genes Dev. 19(11), 12881293. https://doi.org/10.1101/gad.1310605 (2005).

33. Huang, J. et al. A zebrafish model of conditional targeted podocyte ablation and regeneration. Kidney Int. 83(6), 1193-1200. https:// doi.org/10.1038/ki.2013.6 (2013).

34. Siegerist, F., Zhou, W., Endlich, K. \& Endlich, N. 4D in vivo imaging of glomerular barrier function in a zebrafish podocyte injury model. Acta Physiol. (Oxf). 220(1), 167-173. https://doi.org/10.1111/apha.12754 (2017).

35. Lin, C.-Y. et al. MiR-1 and miR-206 target different genes to have opposing roles during angiogenesis in zebrafish embryos. Nat. Commun. 4, 2829. https://doi.org/10.1038/ncomms3829 (2013).

36. Agrawal, R., Tran, U. \& Wessely, O. The miR-30 miRNA family regulates Xenopus pronephros development and targets the transcription factor Xlim1/Lhx1. Development 136(23), 3927-3936. https://doi.org/10.1242/dev.037432 (2009).

37. Wienholds, E. et al. MicroRNA expression in zebrafish embryonic development. Science 309(5732), 310-311. https://doi.org/10. 1126/science.1114519 (2005).

38. Shen, J., Wang, Q., Gurvich, I., Remotti, H. \& Santella, R. M. Evaluating normalization approaches for the better identification of aberrant microRNAs associated with hepatocellular carcinoma. Hepatoma Res. 2, 305-315. https://doi.org/10.20517/2394-5079. 2016.28 (2016).

39. Kloosterman, W. P. et al. Cloning and expression of new microRNAs from zebrafish. Nucleic Acids Res. 34(9), 2558-2569. https:// doi.org/10.1093/nar/gkl278 (2006).

40. Li, N., Wei, C., Olena, A. F. \& Patton, J. G. Regulation of endoderm formation and left-right asymmetry by miR-92 during early zebrafish development. Development 138(9), 1817-1826. https://doi.org/10.1242/dev.056697 (2011). 


\section{Acknowledgements}

This work was funded by ERA-EDTA long term fellowship LTF RLTF 1593/2018, Bundesministerium für Bildung und Forschung (Grant nos. 01GM1518B, STOP FSGS) by N.E., Südmeyer-Stiftung für Nieren- und Gefäßforschung.

\section{Author contributions}

F.S. and T.L. contributed equally. F.S., T.L., and N.E. conceptualized the study and designed experiments. F.S., T.L., A.I., and T.M.K. performed experiments. W.Z. established and contributed transgenic zebrafish models. F.S. and T.L. wrote the main manuscript text and prepared figures. G.C., K.E., and N.E. supervised the study. All authors reviewed and approved the final version of the manuscript.

\section{Funding}

Open Access funding enabled and organized by Projekt DEAL.

\section{Competing interests}

The authors declare no competing interests.

\section{Additional information}

Supplementary Information The online version contains supplementary material available at https://doi.org/ 10.1038/s41598-021-00075-2.

Correspondence and requests for materials should be addressed to N.E.

Reprints and permissions information is available at www.nature.com/reprints.

Publisher's note Springer Nature remains neutral with regard to jurisdictional claims in published maps and institutional affiliations.

(c) (i) Open Access This article is licensed under a Creative Commons Attribution 4.0 International cc) License, which permits use, sharing, adaptation, distribution and reproduction in any medium or format, as long as you give appropriate credit to the original author(s) and the source, provide a link to the Creative Commons licence, and indicate if changes were made. The images or other third party material in this article are included in the article's Creative Commons licence, unless indicated otherwise in a credit line to the material. If material is not included in the article's Creative Commons licence and your intended use is not permitted by statutory regulation or exceeds the permitted use, you will need to obtain permission directly from the copyright holder. To view a copy of this licence, visit http://creativecommons.org/licenses/by/4.0/.

(C) The Author(s) 2021 